\title{
Psychotropic Medication Use among Medicare Beneficiaries Following Traumatic Brain Injury
}

Jennifer S. Albrecht ${ }^{1}$, PhD, Daniel C. Mullins ${ }^{2}, \mathrm{PhD}$, Gordon S. Smith ${ }^{1,3}, \mathrm{MB}, \mathrm{ChB}, \mathrm{MPH}$, Vani $\mathrm{RaO}^{4}, \mathrm{MD}$

${ }^{1}$ Department of Epidemiology and Public Health, University of Maryland School of Medicine

${ }^{2}$ Department of Pharmaceutical Health Services Research, University of Maryland School of Pharmacy

${ }^{3}$ Shock, Trauma and Anesthesiology Research (STAR) - Organized Research Center, National Study Center for Trauma and Emergency Medical Services, University of Maryland

${ }^{4}$ Department of Psychiatry \& Behavioral Sciences, Johns Hopkins University School of Medicine

Corresponding Author: Dr. Jennifer S. Albrecht, Department of Epidemiology and Public Health, University of Maryland School of Medicine, MSTF 334C, 10 S. Pine St., Baltimore, MD 21201, email: jalbrecht@epi.umaryland.edu, phone: 410-706-0071, fax: 410-706-0098

Conflicts of Interest and Source of Funding: Dr. Albrecht was supported by AHRQ grant 1K01HS024560. Dr. Smith was supported by NIH grant R01AA18707. Dr. Rao was supported by DOD grant W81XWH-13-1-0469. The authors declare no conflicts of interest.

Portions of these results were presented at the 11th World Congress on Brain Injury. The Hague, Netherlands, March, 2016. 
Abstract

Objectives: To characterize psychotropic medication use before and after traumatic brain injury (TBI) hospitalization among older adults. A secondary objective is to determine how receipt of indicated pharmacologic treatment for anxiety and post-traumatic stress disorder (PTSD) differs following TBI.

Design: Retrospective cohort

Setting: United States

Participants: Medicare beneficiaries age $\geq 65$ hospitalized with TBI 2006-2010 with continuous drug coverage for 12 months before and after TBI $(n=60,276)$.

Measurements: We obtained monthly psychotropic medication use by drug class and specific drugs from Medicare Part D drug event files. International Classification of Disease, $9^{\text {th }}$ Edition $\mathrm{CM}$, codes were used to define anxiety (300.0x) and PTSD (309.81).

Results: Average monthly prevalence of psychotropic medication use among all patients hospitalized for TBI was 44.8\%; antidepressants comprised 73\%. Prevalence of psychotropic medication use increased from 2006-2010. Following TBI, psychotropic medication use increased slightly (odds ratio (OR) 1.05; 95\% confidence interval (CI) 1.03, 1.06.) Tricyclic antidepressant use decreased post-TBI (OR $0.76 ; 95 \% \mathrm{Cl} 0.73,0.79)$ while use of the sedating antidepressants mirtazapine (OR $1.31 ; 95 \% \mathrm{Cl} 1.25,1.37)$ and trazadone (OR $1.11 ; 95 \% \mathrm{Cl} 1.06$, 1.17) increased. Antipsychotic (OR 1.15; 95\% $\mathrm{Cl} 1.12,1.19$ ) use also increased post-TBI. Beneficiaries newly diagnosed with anxiety (OR $0.42 ; 95 \% \mathrm{Cl} 0.36,0.48)$ and/or PTSD (OR 0.39; $95 \% \mathrm{Cl} 0.18,0.84)$ post-TBI were less likely to receive indicated pharmacologic treatment. 
Conclusions: Older adults hospitalized with TBI have a high prevalence of psychotropic medication use yet are less likely to receive indicated pharmacological treatment for newly diagnosed anxiety and PTSD following TBI. 
Introduction

Traumatic brain injury (TBI) causes significant morbidity among older adults, resulting in over 142,000 emergency department visits and 81,500 hospitalizations annually in the United States. ${ }^{1}$ Further, rates of TBI among older adults are increasing. ${ }^{1,2}$ Older adults have the highest rates of hospitalization and mortality following $\mathrm{TBI}$ and are more likely to experience poor cognitive and functional outcomes, nursing home placement, and mortality compared to younger adults with similar injury severity. ${ }^{1,3-6}$

Individuals with TBI are at increased risk of neuropsychiatric disorders due to 1) trauma to brain regions involved in emotional regulation and behavioral control, and 2) longer-term cognitive and physical disability from the TBI resulting in loss of employment, previously enjoyed activities, family support, and social contacts. ${ }^{7-10}$ Although there is good evidence of the increased risk of neuropsychiatric disorders following TBI, there is insufficient high-level evidence supporting the use of any pharmacologic treatments for neuropsychiatric disorders following TBI. ${ }^{10,11}$ Brain injury increases the risk of adverse events such as seizures and stroke, raising concerns that treatments for neuropsychiatric disorders may not have the same safety or efficacy in individuals with TBI. ${ }^{11-15}$ Physicians currently rely on evidence from a few small clinical trials and treatment of neuropsychiatric disturbances in the general population to guide treatment decisions for individuals with TBI. ${ }^{8,11,12,16-20}$

Preliminary to the conduct of comparative safety analyses, a thorough examination of current use of psychotropic medications among individuals with TBI is warranted. Hence, the objective of this study is to characterize psychotropic medication use before and after TBI 
among older adults, a vulnerable population for whom there is little data on treatment of TBI neuropsychiatric disorders. As a secondary objective, we examine whether receipt of indicated pharmacologic treatment for anxiety and post-traumatic stress disorder (PTSD) is impacted by TBI.

Methods

We conducted a retrospective analysis of Medicare administrative claims data obtained from the Centers for Medicare \& Medicaid Services (CMS) Chronic Condition Data Warehouse (CCW). In the United States, Medicare provides health insurance to individuals aged 65 and older and younger individuals with disabling conditions. Medicare consists of three Parts: A (hospital), B (medical), and D (outpatient prescription medications). Beneficiaries can also enroll in Part C, in which a commercial insurer provides benefits. All elderly Medicare beneficiaries hospitalized with TBI during 2006-2010 were eligible. Inclusion criteria were age $\geq 65$ at TBI, first TBI, and survival to hospital discharge. We defined TBI by searching for International Classification of Disease, 9th Revision Clinical Modification (ICD-9-CM) codes 800.xx, 801.xx, 803.xx, 804.xx, 850.xx- 854.1x, 950.1-950.3, 959.01) in any position on an inpatient claim. These

ICD-9-CM codes represent the Centers for Disease Control and Prevention's (CDC) case definition for TBI, which previously has been reported to have a sensitivity of $89 \%$ to detect severe TBI and a positive predictive value of $93 \%$ to correctly identify TBI-related hospitalizations. ${ }^{21,22}$

Psychotropic Medications 
We searched Medicare Part D prescription drug event files for evidence of psychotropic medication use. We created 30-day periods pre- and post-TBI hospitalization and defined medication use as 1) a filled prescription for any psychotropic medication; or 2) a proportion of days covered (number of daily doses in the prescription/number of days in the month) for any psychotropic medication $>0$. We defined five classes of psychotropic medications as:

antidepressants, antipsychotics, mood stabilizers and anti-seizure medications, other anxiolytics (e.g. buspirone, hydrozyzine), and miscellaneous psychotropics (e.g. zolpidem, zaleplon). Appendix 1 contains a complete list of drug names, classes to which they were assigned, and indicated use in treatment of anxiety of PTSD. To ensure adequate time to capture patterns of medication use as well as comorbidities and diagnosis of neuropsychiatric disturbances, we required twelve months continuous Medicare Parts A, B, and D with no Part C (Medicare Advantage (HMO)) coverage both pre- and post-TBI hospitalization (twenty-four months total follow-up). Medications used during the hospitalization episode and during a skilled nursing facility (SNF) stay of $<100$ days are not consistently present in the Part D drug event file.

\section{Anxiety and PTSD}

Anxiety disorder (henceforth called anxiety) was defined as the presence of ICD-9-CM codes 300.0x on any inpatient or outpatient claim during the study period and PTSD as ICD-9CM code 309.81. The first date of diagnosis occurring pre- and post-TBI was recorded and used to identify individuals with pre- or post-TBI disorders. We also created a variable indicating a new diagnosis post-TBI for those who did not have a previous anxiety or PTSD diagnosis.

\section{Covariates}


Demographic and enrollment characteristics were obtained from the CCW enrollment file and baseline comorbidities at TBI hospitalization were determined using CMS's CCW flagged comorbid conditions. ${ }^{23}$ These 27 chronic conditions are identified based on the presence of ICD9 codes on inpatient, skilled nursing, home health, or outpatient claims using algorithms defined by CMS. The date of first diagnosis is listed for each flagged condition. We used these to create time-varying comorbidity diagnoses throughout the study period.

Diagnosis and treatment of depressive disorder (depression) could strongly influence receipt of treatment for anxiety or PTSD, especially since medications used to treat these conditions often overlap. Therefore, we created indicator variables for pre- and post-TBI depression diagnoses, defined as the presence of ICD-9-CM codes 296.2, 296.3, 300.4, and 311 on any inpatient or outpatient claim during the study period. We also created a variable indicating new diagnosis of depression post-TBI for those without a depression diagnosis preTBI.

Data Analysis

Distributions of demographic and clinical variables in the study population were assessed overall and stratified by receipt of at least one psychotropic medication at any time during the study period. Average monthly prevalence of psychotropic use was calculated by category for the twelve months prior to and following TBI hospitalization. Antidepressants comprise the majority of psychotropic use; therefore we also calculated the average monthly prevalence of use of the most common antidepressants: selective serotonin re-uptake inhibitors (SSRIs), serotonin-norepinephrine reuptake inhibitors (SNRIs), and tri-cyclic 
antidepressants (TCAs). The 'other' antidepressants included mirtazapine, bupropion, trazadone, and monoamine oxidase inhibitors. We also calculated average prevalence of use for the twenty most prevalent individual psychotropic medications during the pre- and post-TBI periods. Finally, we created an indicator variable for use of more than one psychotropic medication class at one time.

To test time trends in monthly prevalence of psychotropic use over the study period, we used linear regression, evaluating trends prior to and following the TBI hospitalization separately. We also evaluated trends over the entire study period, excluding the first two months following injury because of incomplete reporting of medication use during hospitalization and subsequent skilled nursing facility stay. These models were adjusted only for time, and informed adjustment of generalized estimating equation models. To test whether TBI hospitalization impacted psychotropic use, we used generalized estimating equations with a logistic model, accounting for repeated observations and time. As before, we excluded the first two months following injury.

We examined whether individuals newly diagnosed with anxiety or PTSD following TBI used the same amount of psychotropic medication compared to individuals without TBI. However, Medicare beneficiaries hospitalized with TBI differ from other Medicare beneficiaries in that they are older with a greater burden of comorbid illness, complicating efforts to identify a suitable comparison group. ${ }^{24}$ Our prior study examining post-TBI treatment of depression addressed this issue by using the pre-TBI period as the control. ${ }^{25}$ We used similar methodology to compare receipt of treatment for anxiety and PTSD during the pre- and post-TBI periods. 
To focus on new use of psychotropic medications associated with diagnosis of anxiety or PTSD, we excluded beneficiaries with any use of medications used to treat anxiety or PTSD (depending on the model) during the six months prior to diagnosis. To accurately report use of psychotropic medications associated with diagnosis of anxiety or PTSD at the monthly level, we created time-varying indicators for new diagnoses using dates of first ICD-9 CM code for anxiety disorder or PTSD during the pre- and post-TBI period. For this analysis, we compared receipt of treatment pre- vs post-TBI among beneficiaries with new anxiety or PTSD diagnoses. The preand post-TBI groups were mutually exclusive, but an individual with both anxiety and PTSD could contribute follow-up time to both cohorts. We used generalized estimated equations with a logistic model to estimate the odds of psychotropic medication receipt pre- vs post-TBI among beneficiaries newly diagnosed with anxiety or PTSD. Covariates were selected for our models based on bivariate analysis (chi-square or Student's t-test $p$-value $<0.001$ ), a chi-square p-value $<0.05$ from type-III analysis, and a $10 \%$ or greater change in the effect measure parameter estimate.

This study was approved by the Institutional Review Board of the University of Maryland, Baltimore. All analysis was performed with SAS version 9.4 (SAS Institute, Cary, NC).

Results

There were 60,276 beneficiaries meeting inclusion criteria with twelve months continuous Medicare Parts A, B, and D with no Part C coverage before and after TBI hospitalization. Of these, $42,608(71 \%)$ used a psychotropic medication at least once during the twenty-four month study period (Table 1). Beneficiaries who used any psychotropic medication 
were younger (80.4 (standard deviation (sd) 8.3) years vs. 81.1 (sd 8.3) years, Student's t-test, Satterthwaite method $\mathrm{df}=32,614 \mathrm{p}<0.001)$ and more likely to be female ( $71 \%$ vs. $60 \%$, Pearson's $\left.X^{2}=582.2, p<0.001\right)$. They were more likely to have Alzheimer's disease and related dementias ( $24 \%$ vs. $19 \%$, Pearson's $X^{2}=2,548.7, p<0.001$ ) and to have originally qualified for Medicare benefits due to disability rather than age $\left(14 \%\right.$ vs. $8 \%$, Pearson's $\left.X^{2}=423.6, p<0.001\right)$. Among beneficiaries who used psychotropic medications, $27 \%$ were diagnosed with depression pre-TBI and $34 \%$ post-TBI, with $18 \%$ receiving first-time diagnoses post-TBI. Anxiety was diagnosed among $14 \%$ pre-TBI and $17 \%$ post-TBI, and $10 \%$ received a first-time diagnosis post-TBI. PTSD was rarely diagnosed both pre- and post-TBI $(<1 \%)$.

Average monthly prevalence of psychotropic medication use during the study period was $44.8 \%$ (Table 2). Prevalence of use of two or more psychotropic medications at the same time was $14 \%$. Antidepressants were the most commonly used class of medications, with average monthly prevalence of $32.5 \%$ and of these, SSRIs were the most common $(22.2 \%$ average monthly prevalence). There was a significant time trend in use of psychotropic medications over the entire study period (Student's t-test $d f=21, p<0.001$ )(Figure 1), even when month three was excluded to account for incomplete reporting of medication use during hospitalization and subsequent skilled nursing facility stay. Increased use over the study period was observed for all psychotropics except TCAs (significant decrease in use, Student's t-test df $=21, p<0.001$ ) and miscellaneous psychotropics (Student's t-test $d f=21, p=0.12$ ).

The SSRIs sertraline and escitalopram were the most commonly used psychotropic medications before and after TBI (Figure 2). Quetiapine was the most commonly used 
antipsychotic both pre- and post-TBI. Prevalence of use of the anti-seizure agent levetiracetam was low pre-TBI but increased five-fold post-TBI.

Accounting for repeated observations and time, odds of using any psychotropic medication were increased post-TBI (odds ratio (OR) 1.05; 95\% confidence interval (CI) 1.03, 1.06, Wald $X^{2}=36.2, d f=1, p<0.001$ ) (Table 2). Overall, antidepressant use decreased slightly post-TBI, with TCAs showing the largest decrease in use (OR 0.76; $95 \% \mathrm{Cl} 0.73,0.79$, Wald $\left.X^{2}=178.5, d f=1, p<0.001\right)$, even though other antidepressant use increased $(O R 1.15 ; 95 \% \mathrm{Cl}$ 1.11, 1.18, Wald $\left.X^{2}=73.0, d f=1, p<0.001\right)$. The increase in other antidepressant use post-TBI was driven by mirtazapine (OR 1.31; $95 \% \mathrm{Cl} 1.25,1.37$, Wald $\left.\mathrm{X}^{2}=123.3, \mathrm{df}=1, \mathrm{p}<0.001\right)$ and trazadone (OR 1.11; 95\% Cl 1.06, 1.17, Wald $X^{2}=18.3, d f=1, p<0.001$ )(data not shown). Antipsychotic use increased post-TBI (OR 1.15; 95\% Cl 1.12, 1.19, Wald $X^{2}=103.0, \mathrm{df}=1$, $p<0.001)$, driven by $15 \%-20 \%$ increases in the atypical antipsychotics aripiprazole, quetiapine, olanzapine, and risperidone (data not shown). Mood stabilizer/anti-seizure use increased significantly post-TBI (OR 1.21; 95\% Cl 1.19, 1.24, Wald $\left.X^{2}=250.8, d f=1, p<0.001\right)$. The increase was largest for the anti-seizure drug levetiracetam (OR 4.80; $95 \% \mathrm{Cl} 4.37,5.28$, Wald $\left.X^{2}=1,066.5, d f=1, p<0.001\right)$ (data not shown).

There were 3,402 beneficiaries diagnosed with anxiety pre-TBI with no use of indicated psychotropic medications during the six months prior to diagnosis and 1,676 beneficiaries diagnosed with anxiety for the first time post-TBI and with no use of indicated psychotropic medications for at least six-months prior to anxiety diagnosis, for a total of 5,078 beneficiaries. Our adjusted model contained terms for TBI, age, sex, race, calendar year, Alzheimer's disease 
and related dementias, pre-TBI diagnosis of depression and post-TBI diagnosis of depression (these variables were time-varying). Beneficiaries newly diagnosed with anxiety post-TBI were less likely to receive indicated pharmacologic treatment (OR 0.42; 95\% $\mathrm{Cl} 0.36,0.48$, Wald $\left.X^{2}=145.5, d f=1, p<0.001\right)($ Table 3).

There were 51 beneficiaries diagnosed with PTSD pre-TBI with no use of indicated psychotropic medications during the six months prior to diagnosis and 34 beneficiaries diagnosed with PTSD for the first time post-TBI with no use of indicated psychotropic medications for at least six months prior to PTSD diagnosis, for a total of 85 beneficiaries. Our adjusted model contained terms for TBI and original reason for Medicare entitlement (age, disability, end-stage renal disease). Beneficiaries newly diagnosed with PTSD post-TBI were less likely to receive indicated pharmacologic treatment $\left(\mathrm{OR} 0.39 ; 95 \% \mathrm{Cl} 0.18,0.84\right.$, Wald $\mathrm{X}^{2}=5.8$, $d f=1, p=0.02)($ Table 3).

Discussion

In this national study of Medicare beneficiaries hospitalized with TBI, almost threequarters used psychotropic medications at least once and monthly prevalence of use increased over the $\mathbf{2 4}$ month study period. The majority of those using psychotropic medications did not receive a diagnosis of depression, anxiety, or PTSD during the study period. After accounting for repeated measures and time, hospitalization for TBI resulted in decreases in antidepressant use after discharge while use of mood stabilizers/anti-seizure medications and antipsychotics increased. Compared to individuals diagnosed with anxiety or PTSD pre-TBI, those diagnosed post-TBI were much less likely to receive indicated pharmacological treatment. 
Although information on prevalence of psychotropic use is limited, prevalence of antidepressant use (33\%) in our study was much higher than prior estimates. Kantor et al. (2015) assessed antidepressant use among participants $\geq 18$ years in the National Health and Nutrition Examination Survey and reported that during 2007-2010, 12\% of study participants reported using an antidepressant in the past 30 days. ${ }^{26}$ Depression has been identified as a potential risk factor for $\mathrm{TBI}$, hence our sample may have been enriched for individuals with greater antidepressant use. ${ }^{27,28}$ Consistent with this hypothesis, the sample population was majority female and had a high prevalence of Alzheimer's, two factors associated with depression. ${ }^{29-31}$ Additionally, there is some evidence of an association between SSRIs and increased risk of falls and fractures, suggesting that we may be observing a risk factor for TBI, although our study did not examine this question..$^{32,33}$

The SSRIs sertraline and escitalopram were the most commonly prescribed psychotropic medications both pre- and post-TBI, consistent with results of a previous study conducted among older adults and with currently used treatments for depression following TBI. ${ }^{8,11,34}$ Odds of TCA use, which can lower the seizure threshold and is associated with increased risk of metabolic and anticholinergic side-effects, decreased $24 \%$ compared with the pre-TBI period. ${ }^{8,11}$ This large decrease suggests prescriber awareness of potential adverse events associated with TCA use following TBI that may be leading to either discontinuation or switching from TCAs to a different psychotropic medication. There was an overall small but significant decrease in antidepressant use not completely attributable to TCAs that supports discontinuation following TBI and may reflect caution on the part of providers to continue prescribing antidepressants, perhaps due to a desire to decrease polypharmacy or possibly 
knowledge that certain antidepressants are associated with increased fall risk. Use of the sedating antidepressants trazadone and mirtazapine increased moderately ( $15 \%$ increase in odds of use) post-TBI, likely resulting from treatment of sleep disturbances, common following TBI. ${ }^{8}$ Finally, odds of use of atypical antipsychotics, among which quetiapine is most commonly used, increased by a moderate $15 \%$ post-TBI. Quetiapine, and to a lesser extent risperidone, is often used for the treatment of insomnia, agitation, and aggression following TBI. ${ }^{8}$

Individuals newly diagnosed with anxiety and PTSD post-TBI were much less likely to receive indicated medications for treatment of these disorders compared to similar individuals diagnosed with anxiety and PTSD before TBI. This finding is consistent with our previous results on treatment of depression following TBI and provides evidence that among older adults, there is a disparity in receipt of treatment for the three most common neuropsychiatric disorders following $\mathrm{TBI}^{25}$ Older adults are more likely to initially consult with a primary care provider for psychological problems and receive a psychiatric diagnosis from a non-specialty setting. ${ }^{32}$ However, these providers may not feel comfortable prescribing psychotropic medications following TBI in the absence of evidence based recommendations. ${ }^{11,12,35}$ The disparity may also result from the perception that a psychological reaction to trauma is expected and will resolve on its own without treatment. Cognitive impairment, common following TBI, may also impede filling prescribed medications or following up with providers for refills. ${ }^{36}$

Limitations of this study include lack of a comparison group of Medicare beneficiaries who were not hospitalized with TBI. Although we used the pre-TBI period for comparison, it is likely that psychotropic medication use in this population is different from and possibly higher 
than that of other Medicare beneficiaries. Information on TBI severity is missing from administrative claims; however, neuropsychiatric disturbances occur regardless of TBI severity and current pharmacologic treatments do not account for severity of injury. ${ }^{8,11}$ Furthermore, this study includes only beneficiaries hospitalized with TBI. These individuals may have been more severely injured or more susceptible to complications compared to those who were treated and released in the emergency department. If these factors were also associated with psychotropic medication use, bias could result. Future studies should focus on different populations to help determine the extent of this potential bias. Additionaly, while we assessed filled prescriptions, we don't have information on medications prescribed but not filled or adherence to filled prescriptions. Therefore, observed differences in psychotropic medication use pre-post TBI may be due to lack of prescribing or lack of follow-up on the part of the patient. Finally, we don't know the indications for which beneficiaries were receiving psychotropic medications.

Nonetheless, this is the largest study of psychotropic medication use among individuals with TBI and it provides critical information on current usage patterns before and after TBI hospitalization. Comparative safety and effectiveness analyses could initially target the medications we identified as most often used. Furthermore, this study highlights a disparity in receipt of pharmacological treatment for newly diagnosed anxiety and PTSD following TBI. Future studies should focus on elucidated underlying reasons for the observed disparity and whether younger adults are equally affected. More research is also needed to determine the efficacy of psychotropic medications following TBI, their specific side-effects and drug-drug interactions and the role of non-pharmacological strategies. Physicians should be cognizant of 
these factors and be extremely cautious in prescribing psychotropics in elderly individuals with TBI who are often being treated pharmacologically for other medical conditions.

In conclusion, Medicare beneficiaries hospitalized with TBI have a high prevalence of psychotropic medication use both before and after TBI yet are less likely to receive indicated pharmacological treatment for newly diagnosed anxiety and PTSD following TBI. 


\section{References}

1. Faul M, Xu L, Wald MM, et al. Traumatic Brain Injury in The United States: Emergency Department Visits, Hospitalizations, and Deaths. Atlanta (GA): Centers for Disease Control and Prevention, National Center for Injury Prevention and Control; 2010.

2. Albrecht JS, Hirshon JM, McCunn M, et al. Increased Rates of Mild Traumatic Brain Injury Among Older Adults in US Emergency Departments, 2009-2010. J Head Trauma Rehabil. 2015;Oct 16. [Epub ahead of print]

3. Thompson HJ, McCormick WC, Kagan SH. Traumatic Brain Injury in Older Adults: Epidemiology, Outcomes, and Future Implications. J Am Geriatr Soc.2006. 54:1590-1595

4. Harvey LA and Close, JCT. Traumatic Brain Injury in Older Adults: Characteristics, Causes and Consequences. Injury, Int. J. Care Injured. 2012. 43:1821-1826

5. Mosenthal AC, Lavery RF, Addis M, et al. Isolated Traumatic Brain Injury: Age Is an Independent Predictor of Mortality and Early Outcome. J Trauma. 2002. 52:907-911

6. Thompson HJ, Weir S, Rivara FP, et al. Utilization and Costs of Health Care after Geriatric Traumatic Brain Injury. J Neurotrauma. 2012;29:1864-1871

7. Rogers JM, Read CA. Psychiatric Comorbidity Following Traumatic Brain Injury. Brain Inj. $2007 ; 21: 1321-1333$

8. Rao V, Koliatsos V, Ahmed F, et al. Neuropsychiatric Disturbances Associated with Traumatic Brain Injury: A Practical Approach to Evaluation and Management. Semin Neurol 2015;35:64-82.

9. Jorge RE, Arciniegas DB. Mood Disorders after TBI. Psychiatr Clin North Am. 2014;31(1):13- 
10. National Institutes of Health Consensus Development Panel on Rehabilitation of Persons with Traumatic Brain Injury. JAMA. 1999;282:974-983

11. Warden DL, Gordon B, McAllister TW, et al, for the Neurobehavioral Guidelines Working Group. Guidelines for the Pharmacologic Treatment of Neurobehavioral Sequelae of Traumatic Brain Injury. J Neurotrauma. 2006;23:1468-1501

12. Fann JR, Hart T, Schomer KG. Treatment for Depression after Traumatic Brain Injury: A Systematic Review. J Neurotrauma. 2009;26, 2383-2402

13. Povlishock JT \& Katz DI. Update of Neuropathology and Neurological Recovery After Traumatic Brain Injury. J Head Trauma Rehabil. 2005;20(1):76-94.

14. Masel BE, DeWitt DS. Traumatic Brain Injury: A Disease Process, Not an Event. J Neurotrauma. 2010. 27:1529-1540.

15. Yeh CC, Chen TL, Hu CH, Chiu WT, Liao CC. Risk of epilepsy after traumatic brain injury: a retrospective population-based cohort study. J Neurol Neurosurg Psychiatry. 2013. 84:441445.

16. Lee HB, Lyketsos CG, Rao V. Pharmacological Management of the Psychiatric Aspects of Traumatic Brain Injury. Int Rev Psychiatry. 2003;15(4):359-70

17. Fann JR, Uomoto JM, Katon WJ. Sertraline in the Treatment of Major Depression Following Mild Traumatic Brain Injury. J Neuropsychiatry Clin Neurosci. 2000;12(2), 226-232

18. Perino C, Rago R, Cicolini A, et al. Mood and Behavioural Disorders Following Traumatic Brain Injury: Clinical Evaluation and Pharmacological Management. Brain Inj. 2001;15(2), 139-48. 
19. Dinan TG, Mobayed M. Treatment Resistant Depression After Head Injury: A Preliminary Study of Amitriptyline Response. Acta Psychiatr Scand. 1992;85(4), 292-4.

20. Wroblewski BA, Joseph AB, Cornblatt RR. Antidepressant pharmacotherapy and the treatment of depression in patients with severe traumatic brain injury: a controlled, perspective study. J Clin Psychiatry. 1996;57, 582-587

21. Thurman DJ. Guidelines for surveillance of central nervous system injury. National Center for Injury Prevention and Control (U.S.), U.S. Department of Health and Human Services, Public Health Service; 1995

22. Marr A, Coronado V. Central nervous system injury surveillance data submission standards-2002. Atlanta (GA): Centers for Disease Control and Prevention, National Center for Injury Prevention and Control (U.S.), U.S. Department of Health and Human Services, Public Health Service; 2004

23. Centers for Medicare and Medicaid Services Chronic Condition Data Warehouse. Available at: http://www.ccwdata.org/web/guest/condition-categories. Accessed 8/18/16.

24. Centers for Medicare and Medicaid Services. Chronic Conditions among Medicare Beneficiaries, Chartbook, 2012 Edition. Baltimore, MD. 2012. Available at: https://www.cms.gov/research-statistics-data-and-systems/statistics-trends-andreports/chronic-conditions/downloads/2012chartbook.pdf. Accessed 8/18/16.

25. Albrecht JS, Kiptanui Z, Tsang Y, et al. Patterns of Depression Treatment in Medicare Beneficiaries with Depression after Traumatic Brain Injury. J Neurotrauma. 2015;32(16):1223-9 
26. Kantor ED, Rehm CD, Haas JS, et al. Trends in Prescription Drug Use Among Adults in the United States From 1999-2012. JAMA. 2015;314(17):1818-31.

27. Dams-O'Connor K, Gibbons LE, Landau A, et al. Health Problems Precede Traumatic Brain Injury in Older Adults. J Am Geriatr Soc. 2016;64:844-848

28. Menant JC, Wong AFK, Trollor JN, et al. Depressive Symptoms and Orthostatic Hypotension Are Risk Factors for Unexplained Falls in Community-Living Older People. J Am Geriatr Soc. 2016;64:1073-1078

29. Maust DT, Oslin DW, Marcus SC. Effect of Age on the Profile of Psychotropic Users: Results from the 2010 National Ambulatory Medical Care Survey. J Am Geriatr Soc 2014;62:358364

30. Akincigil A, Olfson M, Walkup JT, et al. Diagnosis and Treatment of Depression in Older Community-Dwelling Adults: 1992-2005. J Am Geriatr Soc. 2011;59:1042-1051

31. Shippee ND, Hall K, Zeglin J, O’Donnell B, Virnig BA. Medication use among Medicare beneficiaries with medical and psychiatric conditions. Medication Use in Medicare. Data Points \# 19 (prepared by the University of Minnesota DEcIDE Center, under Contract No. HHSA29020100013I ). Rockville, MD: Agency for Healthcare Research and Quality. April 2014. AHRQ Publication No. 14-EHC028-EF

32. Marcum ZA, Perera S, Thorpe JM, et al; Health ABC Study. Antidepressant Use and Recurrent Falls in Community-Dwelling Older Adults: Findings From the Health ABC Study. Ann Pharmacother. 2016;50(7):525-33. 
33. Sterke CS, Ziere G, van Beeck EF, et al. Dose-Response Relationship Between Selective Serotonin Re-Uptake Inhibitors and Injurious Falls: A Study in Nursing Home Residents with Dementia. Br J Clin Pharmacol. 2012;73(5):812-20

34. Sanglier T, Saragoussi D, Milea D, et al. Comparing Antidepressant Treatment Patterns in Older and Younger Adults: A Claims Database Analysis. J Am Geriatr Soc. 2011;59:11971205

35. Klap R, Unroe KT, Unützer J. Caring for Mental Illness in the United States: A Focus on Older Adults. Am J Geriatr Psychiatry. 2003;11(5):517-24.

36. Dikmen SS, Corrigan JD, Levin HS, et al. Cognitive Outcome Following Traumatic Brain Injury. J Head Trauma Rehabil. 2009;24(6):430-438 
Figure 1. Monthly Prevalence of Psychotropic Medication Use among Medicare Beneficiaries Hospitalized with Traumatic Brain Injury 2006-2010 During Twelve Months Pre- and Post-TBI, $n=60,276$

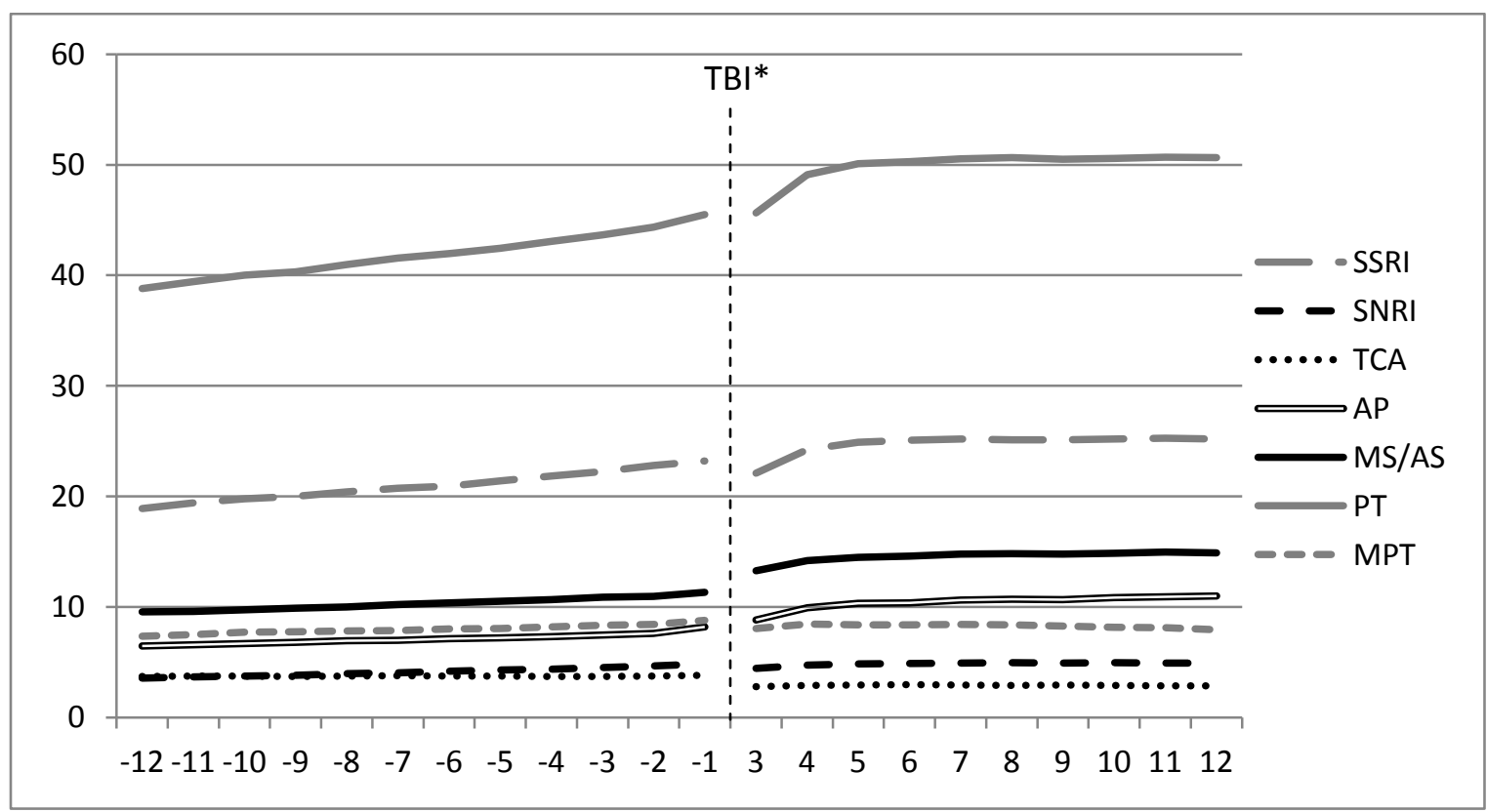

$\mathrm{TBI}$ - traumatic brain injury; SSRI - selective serotonin reuptake inhibitor; SNRI - serotonin norepinephrine reuptake inhibitor; TCA - tricyclic antidepressant; AP - antipsychotic; MS/AS mood stabilizer/anti-seizure; PT - any psychotropic; MPT - miscellaneous psychotropic; *Dip post-TBI is caused by incomplete capture of drug claims data during hospitalization and subsequent skilled nursing facility stay 
Figure 2. Average Monthly Prevalence of the Twenty Most Commonly Used Psychotropic Medications among Medicare Beneficiaries During the Twelve Months Pre- and PostHospitalization with Traumatic Brain Injury 2006-2010, n= 60,276

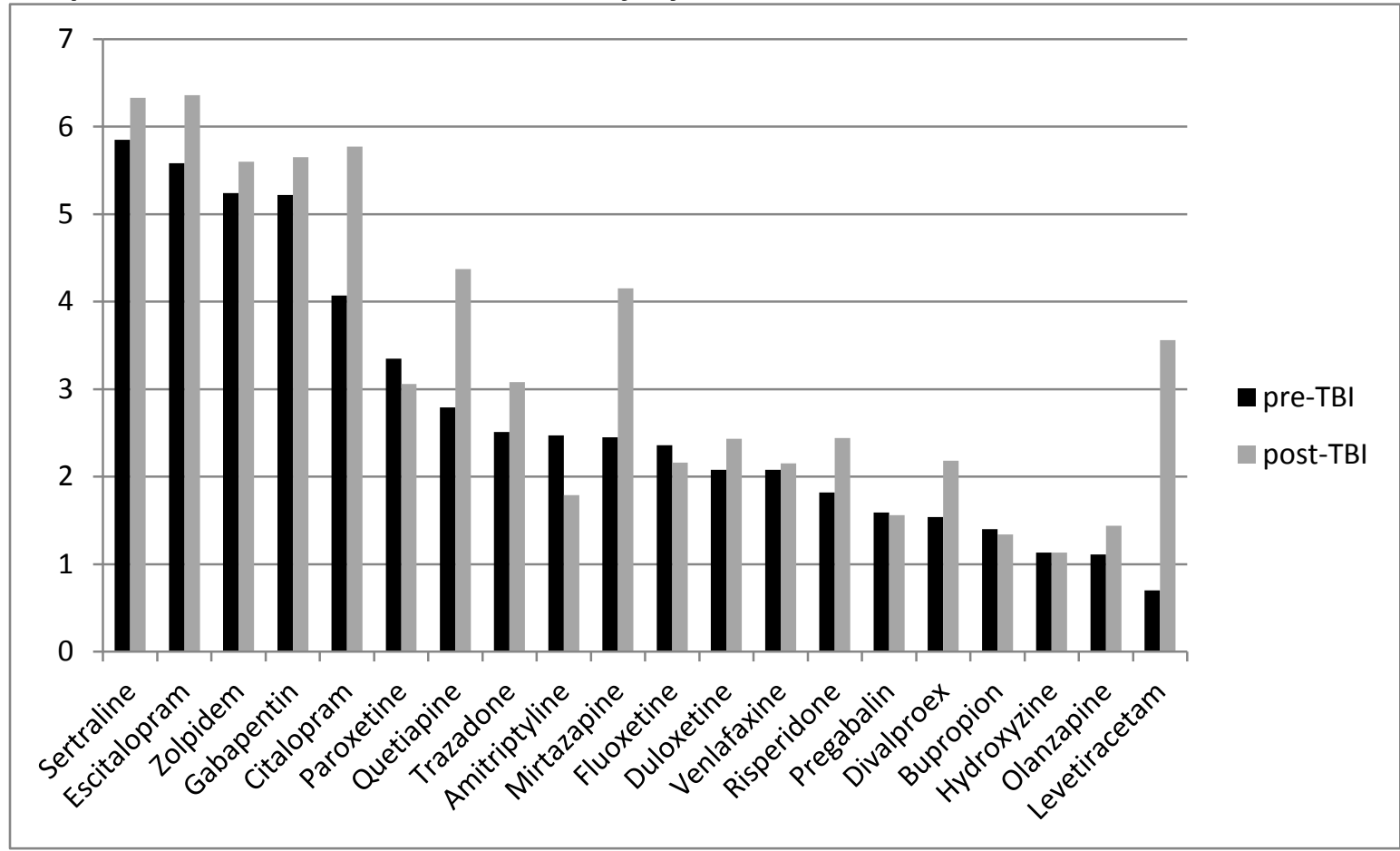


Table 1. Baseline Characteristics of Medicare Beneficiaries Hospitalized with Traumatic Brain Injury 2006-2010 by Receipt of Psychotropic Medications at any Time During the Study Period, $n=60,276$

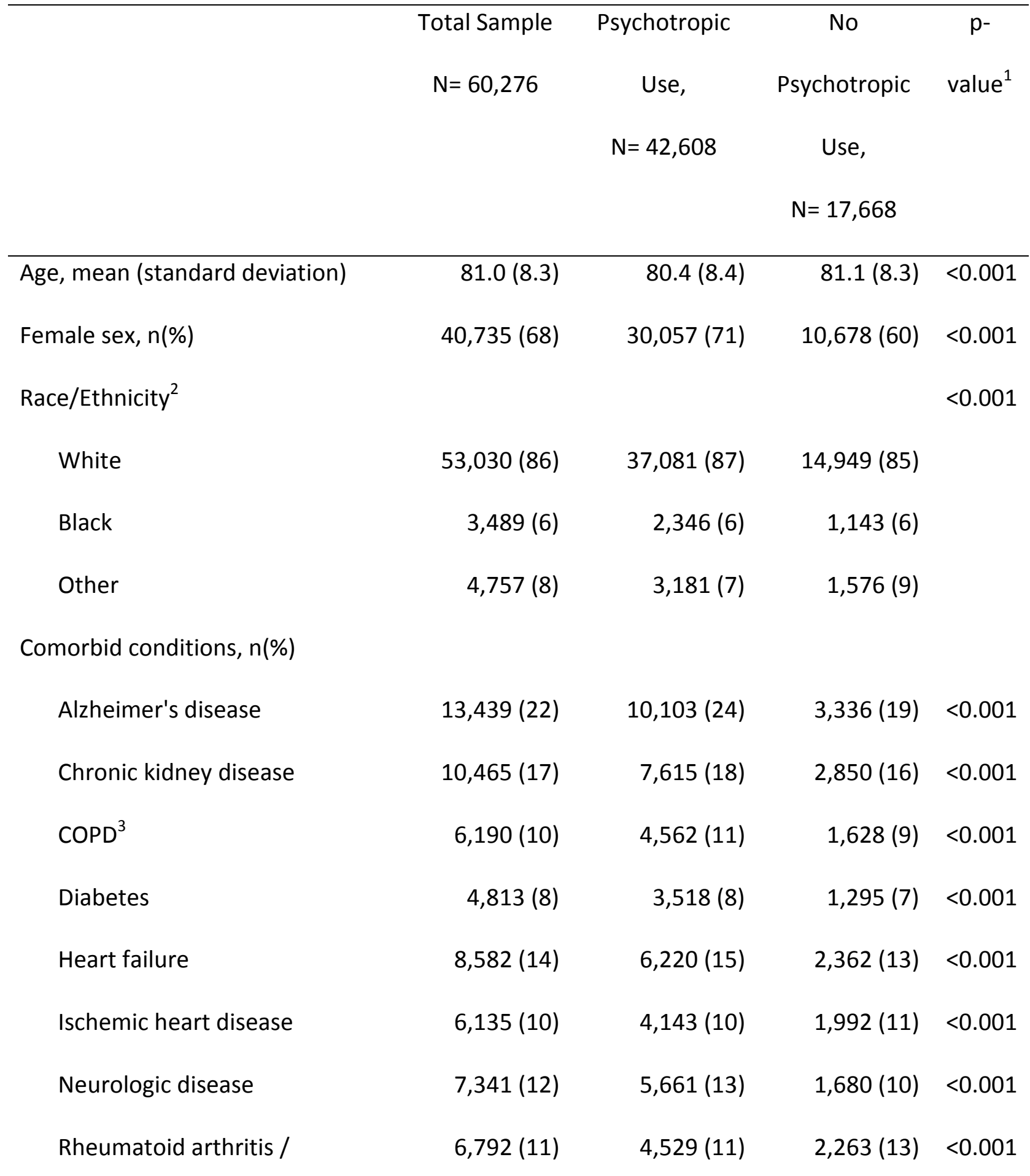

osteoarthritis 
Stroke / transient ischemic

13,664 (23)

$9,804(23)$

$3,860(22)$

0.002

attack

Psychological disorders, n (\%)

Depression pre-TBI

$12,027(20) \quad 11,573(27)$

$454(3)<0.001$

Depression post-TBI

$15,429(26)$

14,692 (34)

$737(4)<0.001$

New depression post-TBI

$8,396(14)$

$7,798(18)$

$598(3)<0.001$

Anxiety pre-TBI

$6,902(11)$

$6,294(14)$

$608(3)<0.001$

Anxiety post-TBI

$8,113(13)$

7,341 (17)

$772(4)<0.001$

New Anxiety post-TBI

5,017 (8)

$4,438(10)$

$579(3)<0.001$

PTSD $^{4}$ pre-TBI

$95(<1)$

$88(<1)$

$7(<1) \quad<0.001$

PTSD $^{4}$ post-TBI

$136(<1)$

$124(<1)$

$12(<1)<0.001$

New PTSD ${ }^{4}$ post-TBI

$102(<1)$

$90(<1)$

$12(<1)<0.001$

OREC $^{2,5}, n(\%)$

$52,823(88)$

$36,584(86)$

16,239 (92)

Age

$7,322(12)$

5,924 (14)

1,398 (8)

$\mathrm{ESRD}^{6}+$-- disability

$131(<1)$

$100(<1)$

$31(<1)$

\footnotetext{
${ }^{1}$ P-value from Pearson's $X^{2}$ (1 degree of freedom except where noted) $=22.1-8,702.3$, Student's t-tests for age (Satterthwaite method $\mathrm{df}=32,614) ;{ }^{2} \mathrm{df}=2 ;{ }^{3}$ Chronic obstructive pulmonary disease; ${ }^{4}$ Post-traumatic stress disorder; ${ }^{5}$ Original reason for Medicare entitlement; ${ }^{6}$ End stage renal disease
} 
Table 2. Average Monthly Prevalence of Psychotropic Medication Use and Odds Ratios Comparing Use Post- to Pre-TBI ${ }^{1}$, Adjusted for Time, among Medicare Beneficiaries Hospitalized with TBI 2006-2010 During Twelve Months Pre- and Post-TBI, n= 60,276

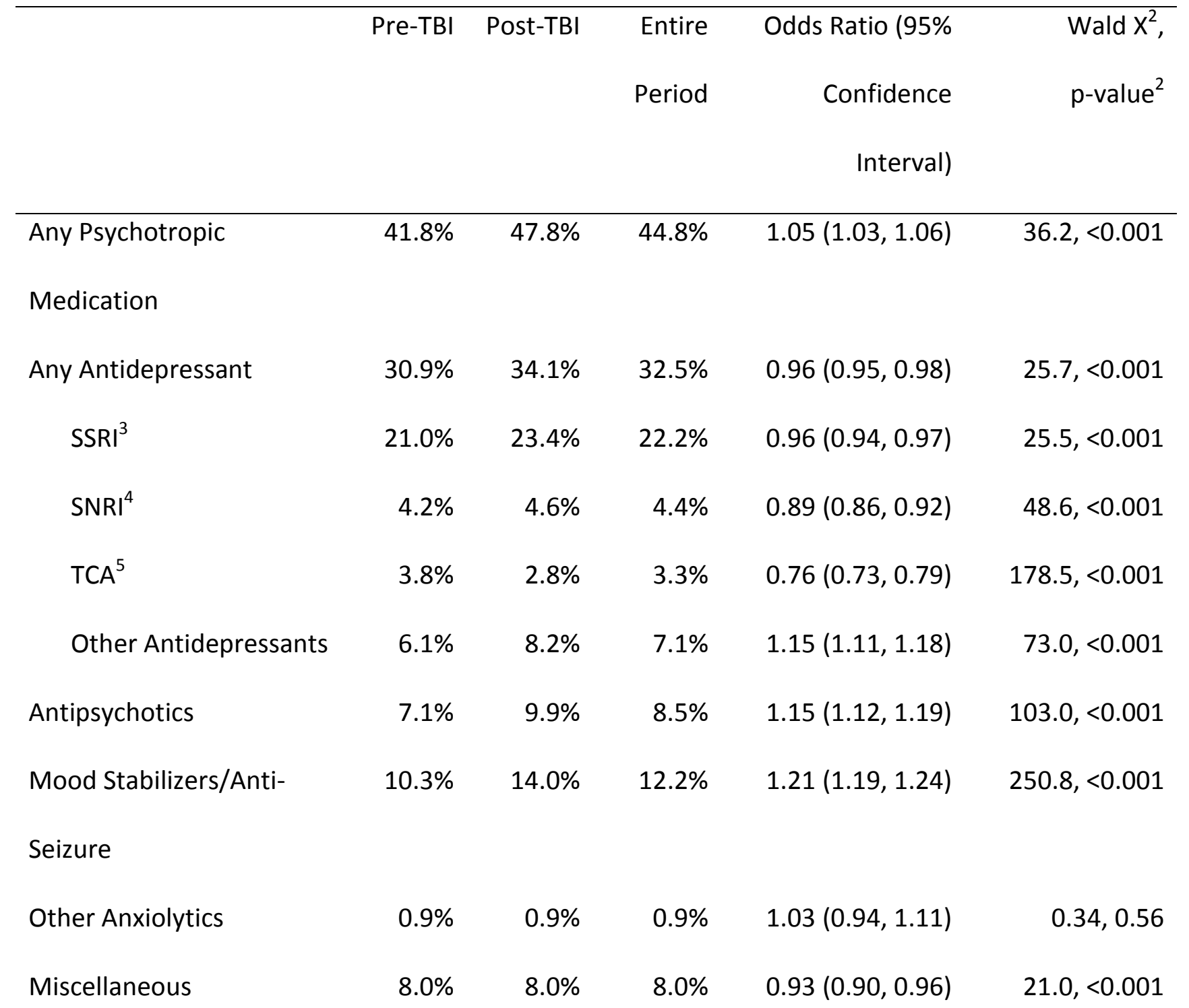

Psychotropics

\footnotetext{
${ }^{1}$ Traumatic brain injury; ${ }^{2}$ Degrees of freedom $=1 ;{ }^{3}$ Selective serotonin reuptake inhibitors;

${ }^{4}$ Serotonin norepinephrine reuptake inhibitors; ${ }^{5}$ Tricyclic antidepressants
} 
Table 3. Among Patients Newly Diagnosed with Anxiety or Post-Traumatic Stress Disorder, Odds of Receiving Indicated Pharmacologic Treatment Post-TBI Compared to Pre-TBI: Medicare Beneficiaries Hospitalized with TBI 2006-2010

Odds Ratio (95\% Confidence Interval) Wald $X^{2}, p$-value

Anxiety, $n=5,078$

Unadjusted

$0.52(0.46,0.58) \quad 126.9,<0.001$

Adjusted $^{1}$

$0.42(0.36,0.48)$

$145.5,<0.001$

Post-traumatic Stress Disorder, $\mathrm{n}=85$

Unadjusted

$0.32(0.15,0.67)$

$9.25,0.002$

Adjusted $^{2}$

$0.39(0.18,0.84)$

$5.8,0.02$

${ }^{1}$ post-TBI, age, sex, race, calendar year, Alzheimer's disease and related dementias, depression pre-TBI, depression post-TBI; ${ }^{2}$ post-TBI, original reason for Medicare entitlement (age, disability, end-stage renal disease) 